\title{
Comparative evaluation of mesenchymal stromal cells from umbilical cord and amniotic membrane in xeno-free conditions
}

\author{
Yongxu $\mathrm{Mu}^{1,2+}$, Xiaoyun $\mathrm{Wu}^{3,4+}$ and Zhiming $\mathrm{Hao}^{1 *}$
}

\begin{abstract}
Background: Within the past years, umbilical cord (UC) and amniotic membrane (AM) expanded in human platelet lysate (PL) have been found to become increasingly candidate of mesenchymal stromal cells (MSCs) in preclinical and clinical studies. Different sources of MSCs have different properties, and lead to different therapeutic applications. However, the similarity and differences between the AMMSCs and UCMSCs in PL remain unclear.

Results: In this study, we conduct a direct head-to-head comparison with regard to biological characteristics (morphology, immunophenotype, self-renewal capacity, and trilineage differentiation potential) and immunosuppression effects of AMMSCs and UCMSCs expanded in PL. Our results indicated that AMMSCs showed similar morphology, immunophenotype, proliferative capacity and colony efficiency with UCMSCs. Moreover, no significantly differences in osteogenic, chondrogenic and adipogenic differentiation potential were observed between the two types of cells. However, AMMSCs exhibited higher PGE 2 expression and IDO activity compared with UCMSCs when primed by IFN- $\gamma$ and (or) TNF-a induction, and AMMSCs showed a higher inhibitory effect on PBMCs proliferation than UCMSCs.

Conclusion: The results suggest that AMMSCs expanded in PL showed similar morphology, immunophenotype, selfrenewal capacity, and trilineage differentiation potential with UCMSCs. However, AMMSCs possessed superior immunosuppression effects in comparison with UCMSCs. These results suggest that AMMSCs in PL might be more suitable than UCMSCs for treatment of immune diseases. This work provides a novel insight into choosing the appropriate source of MSCs for treatment of immune diseases.
\end{abstract}

Keywords: Mesenchymal stromal cells, Amnion, Umbilical cord, Platelet lysate, Characteristics, Immunomodulatory

\section{Background}

Mesenchymal stromal cells (MSCs) are popular cells for regenerative medicine due to their capacity of extensive self-renewal, multilineage differentiation potential, and immunosuppressive effects [1]. Due to their low proportion in human tissues, extensive in vitro expansion is necessary to attain sufficient cell numbers for MSCs-based therapies. Traditionally, culture media for the isolation and expansion of MSCs in basic research and most clinical studies usually comprise fetal bovine serum (FBS).

\footnotetext{
* Correspondence: haozhm66@126.com

${ }^{+}$Yong Xu Mu and Xiao Yun Wu contributed equally to this work.

'Department of Rheumatology, the First Affiliated Hospital of Xi'an Jiaotong

University, Xi'an, Shaanxi Pvovince, China
}

Full list of author information is available at the end of the article
FBS is an animal-derived product, and its usage has raised safety concern [2]. The current regulatory settings aiming to minimize the usage of FBS have reinforced an intensive search for possible substitutes [2]. Over the last decade, many laboratories adapt their "xenogen-free or animal-free" culture condition to human platelet lysate (PL), which allows expansion and clinical grade production of MSCs for clinical applications [3]. The usage of PL in MSCs culture could provide advantages as follows: (1) PL as a human reagent, is the absence of any risk of xenogeneic immune reactions or transmission of bovine pathogens [4]. (2) MSCs in PL-supplemented medium display a smaller in size and more elongated morphology, faster attachment and migration rate, higher

(c) The Author(s). 2018 Open Access This article is distributed under the terms of the Creative Commons Attribution 4.0 International License (http://creativecommons.org/licenses/by/4.0/), which permits unrestricted use, distribution, and reproduction in any medium, provided you give appropriate credit to the original author(s) and the source, provide a link to the Creative Commons license, and indicate if changes were made. The Creative Commons Public Domain Dedication waiver (http://creativecommons.org/publicdomain/zero/1.0/) applies to the data made available in this article, unless otherwise stated. 
proliferation effect, and greater osteogenic and chondrogenic differentiation potential [4]. Therefore, PL has been widely used as a FBS substitute for clinical-scale expansion of MSCs from various sources, although the biological characteristics and (or) therapeutic potential can be changed in PL [5].

MSCs are first identified and isolated from bone marrow, which has emerged as the most common source in MSCs-based therapies and tissue engineering [6]. In recent years, umbilical cord (UC) and amniotic membrane (AM) appear to be more promising sources of MSCs [7]. Both UC and AM are of foetal origin from perinatal tissues, and provide more primitive cells, which exhibit superior cell activity including higher self-renewal capacity, greater differentiation potential and lower immunogenicity when compared with bone marrow [8-10]. Another important advantage of perinatal tissues is that they are usually discarded as a medical waste, can be obtained easily without ethical constraints [8]. Accumulating evidences have shown that UC derived MSCs (UCMSCs) may have a therapeutic advantage for MSCs-based therapies because of their primitive features [11, 12]. Within the past years, AM has been also found to become increasingly candidate of MSCs in preclinical and clinical studies [7].

Besides culture condition, source-dependent differences in biological characteristics of MSCs have recently emerged and lead to different therapeutic applications [13]. The biological characteristics have been compared between UCMSCs and AM derived MSCs (AMMSCs) in FBS-supplemented medium, but the results are in conflict [14-17]. The similarity and differences between both types of cells in PL are not clear to date. Moreover, optimal sources for treatment of immune diseases remain to be identified. In this study we conduct a direct head-to-head comparison with regard to their morphology, immunophenotype, self-renewal capacity, trilineage differentiation potential, and immunosuppressive effects.

\section{Methods}

\section{PL preparation}

PL was prepared from whole blood unit that was harvested from healthy donor between 18 and 65 years old with some modifications as described previously [18]. Briefly, platelet-rich plasma (PRP) was generated by enriching whole blood platelet concentration using a series of centrifugations, and standardized to a concentration of $1 \times 10^{9}$ platelets $/ \mathrm{ml}$ by removing excess platelet-poor plasma (PPP). PL was prepared from PRP by a simple freeze-thawing procedure, centrifuged to remove the platelet fragments, and filtered further using a $40-\mu \mathrm{M}$ filter (BD Biosciences, Franklin Lakes, USA). The preparation methodology is illustrated in Fig. 1a. At least 10 thawed PL were pooled to prepare a standardized pooled PL, and added to heparinized iscove's modified dulbecco's medium (IMDM, $2 \mathrm{U} / \mathrm{ml}$ ).

\section{Quantification of growth factors in PL and PPP}

Several growth factors including platelet-derived growth factor (PDGF), basic fibroblast growth factor (bFGF), epithelial growth factor (EGF), transforming growth factorbeta 1 (TGF- $\beta 1$ ), insulin-like growth factor-1 (IGF-1) and vascular endothelial growth factor (VEGF) have already been known to be required for MSCs growth in vitro, so the quantification of these growth factors in PL and PPP was determined using enzyme linked immunosorbent assay (ELISA) according to the manufacturer's instruction (Sino Biological Inc., Beijing, China).

\section{Human UCMSCs and AMMSCs cultures}

All human UC $(n=5)$ and AM $(n=5)$ samples were obtained from healthy, full-term, complicated pregnancies with informed consent, and the study was approved by the Ethics Committee of the First Affiliated Hospital of Baotou Medical College. UC was sectioned $(5$ to $10 \mathrm{~cm}$ long), and umbilical arteries and vein were removed. The AM was mechanically peeled off from the placenta. Both UC and AM were washed with phosphate buffered saline (PBS) to remove excessive blood and cellular debris, minced into approximately $1 \times 1 \mathrm{~mm}^{3}$ pieces. AM was incubated in $0.25 \%$ trypsin solution for $60 \mathrm{~min}$ in 37 ${ }^{\circ} \mathrm{C}$ to eliminate epithelial cells. An enzyme cocktail (hyaluronidase $5 \mathrm{U} / \mathrm{ml}$, collagenase $125 \mathrm{U} / \mathrm{ml}$ and dispase 50 $\mathrm{U} / \mathrm{ml}$; Sigma, St. Louis, MO, USA) was used to digest the pieces for $60 \mathrm{~min}$ with gentle agitation at $37^{\circ} \mathrm{C}$. The total nucleated cells were plated at a concentration of $2 \times 10^{5} / \mathrm{cm}^{2}$ in $5 \%$ PL-supplemented media. The fresh medium was changed twice per week. When reaching $80 \%$ confluence, cells were replated at $2000 \mathrm{cells} / \mathrm{cm}^{2}$.

\section{Colony-forming unit fibroblast (CFU-F) assays}

Total nucleated cells were plated in six-well culture plates in 5\% PL-supplemented media at densities of $1 \times 10^{5}$ per well. On day 14, the cell layer was fixed with methanol and stained with crystal violet. Individual colonies composed of at least 50 cells were counted.

\section{Proliferation studies}

The population doubling (PD) was determined using the following formula:

$$
P D=\frac{[\log 10(N h)-\log 10(N p)]}{\log 10(2)}
$$

$N_{h}$ is the harvested cells number and $N_{p}$ is the initial cells number. The PD was calculated and added to the $\mathrm{PD}$ of previous passages to generate cumulative population doublings (CPD) of each passage. 


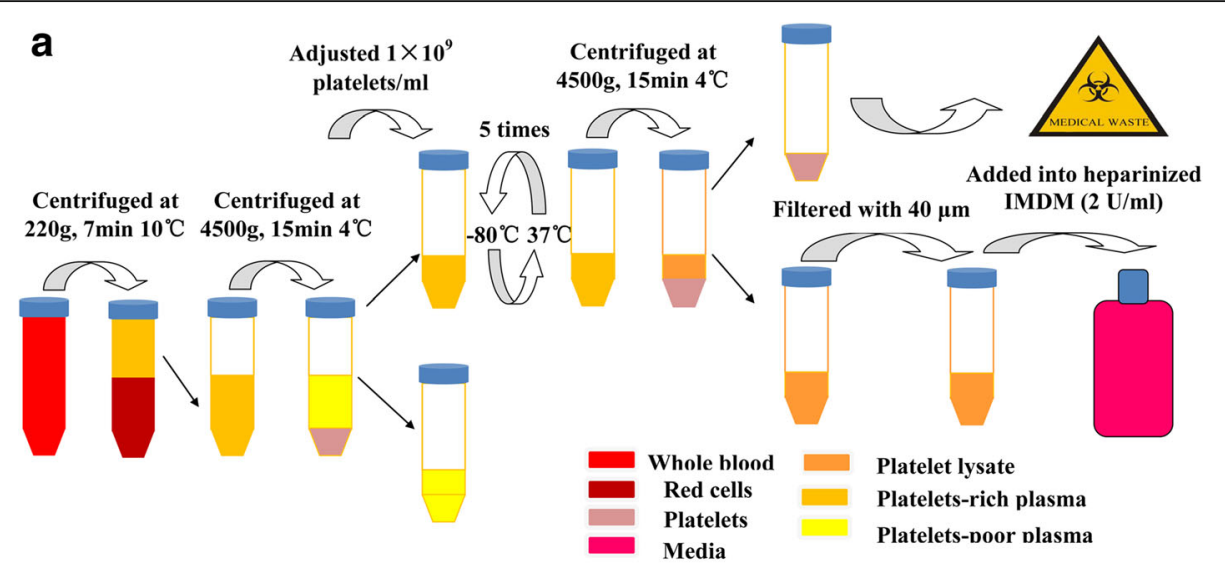

b

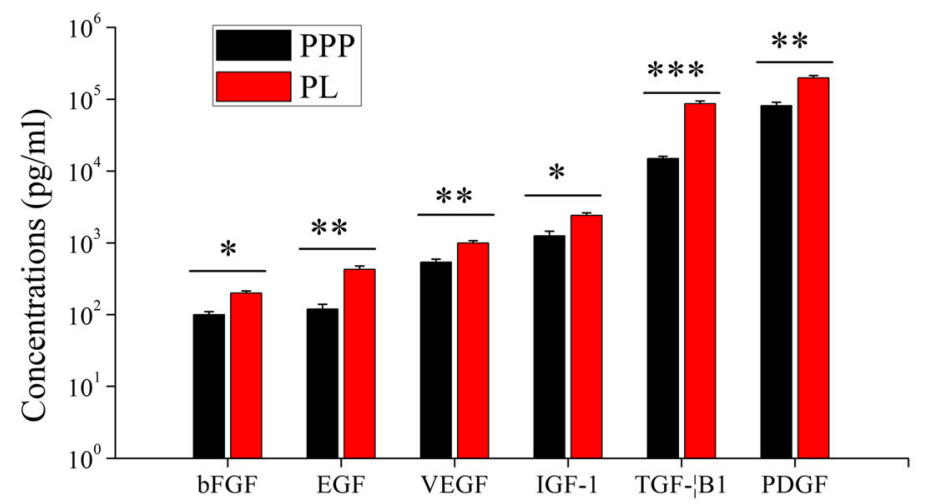

Fig. 1 Platelet lysis released sufficient amounts of growth factors in PL. a Schematic overview of preparation of PL. PRP was generated from peripheral blood through a series of centrifugations. Based on platelet counts $\left(10^{9}\right.$ platelets $\left./ \mathrm{ml}\right)$ after the centrifugation step, sufficient PPP was removed to achieve PRP. PL was prepared from PRP by a simple freeze-thawing procedure. $\mathbf{b}$ The quantification of growth factors in PL and PPP was determined using ELISA. Bars represented means \pm SD. $n=5$; ${ }^{*} p<0.05,{ }^{* *} p<0.01$, and ${ }^{* * *} p<0.005$

\section{Flow cytometry analysis}

Standard flow cytometry analysis was performed to determine the defined MSCs markers for UCMSCs and AMMSCs expanded in PL-supplemented media at passage 5 by using Human MSC Analysis Kit (BD Biosciences, Franklin Lakes, NJ, USA). Data were acquired and analyzed using a FACScan flow cytometer running CellQuest software.

\section{Multilineage differentiation and staining assay}

Osteogenic, chondrogenic and adipogenic differentiation capacity of UCMSCs and AMMSCs was assessed. Both UCMSCs and AMMSCs expanded in PL-supplemented media at passage 5 were induced toward an osteogenic, chondrogenic or adipogenic lineages by using StemPro Osteogenesis, Adipogenesis or Chondrogenesis Differentiation Kit (Gibco, Grand Island, NY, USA), respectively. Osteogenic, chondrogenic and adipogenic differentiation was detected by alizarin red, alcian blue, and oil red $\mathrm{O}$ staining.
Real-time polymerase chain reaction (PCR) analysis

Real-time PCR was performed as described previously [19]. Primers were used in Additional file 1. Platinum SYBR Green qPCR SuperMix-UDG (Invitrogen Darmstadt, Germany) was used under the following conditions: $50^{\circ} \mathrm{Cfor} 2 \mathrm{~min}, 95^{\circ} \mathrm{Cfor} 2 \mathrm{~min}$, and then $95^{\circ} \mathrm{C}$ for $3 \mathrm{~s}$ and $60^{\circ} \mathrm{C}$ for $30 \mathrm{~s}$ for a total of $40 \mathrm{cy}$ cles. Result was analyzed using the $2^{-\Delta \mathrm{Ct}}$ method.

\section{Immune function assay}

Both UCMSCs and AMMSCs cultured in PL-supplemented media at passage 5 were treated with $15 \mathrm{ng} / \mathrm{ml}$ interferon-gamma (IFN- $\gamma$ ) and/or 15 $\mathrm{ng} / \mathrm{ml}$ tumor necrosis factor-alpha (TNF- $\alpha$ ), for $48 \mathrm{~h}$. Prostaglandin $\mathrm{E}_{2}\left(\mathrm{PGE}_{2}\right)$ and TGF- $\beta 1$ concentrations in conditioned medium were quantified using ELISA (Sino Biological Inc., Beijing, China) according to the manufacturer's instructions.: Indoleamine 2, 3-dioxygenase (IDO) activity was evaluated using kynurenine level. 
Table 1 Surface marker expression levels of UCMSCs and AMMSCs in PL

\begin{tabular}{|c|c|c|c|}
\hline \multirow{2}{*}{$\begin{array}{l}\text { Surface } \\
\text { marker }\end{array}$} & \multicolumn{2}{|c|}{ Expression level (\%) } & \multirow{2}{*}{$\begin{array}{l}P \\
\text { value }\end{array}$} \\
\hline & UCMSCS & AMMSCS & \\
\hline CD73 & $96.82 \pm 2.35$ & $97.48 \pm 1.74$ & $P>0.05$ \\
\hline CD90 & $99.24 \pm 1.53$ & $99.76 \pm 1.29$ & $P>0.05$ \\
\hline CD105 & $97.44 \pm 2.08$ & $97.74 \pm 3.28$ & $P>0.05$ \\
\hline CD14 & $1.43 \pm 0.52$ & $1.67 \pm 0.28$ & $P>0.05$ \\
\hline CD19 & $0.96 \pm 0.24$ & $0.75 \pm 0.31$ & $P>0.05$ \\
\hline CD34 & $1.71 \pm 0.60$ & $1.59 \pm 0.53$ & $P>0.05$ \\
\hline CD45 & $1.73 \pm 0.73$ & $1.41 \pm 1.15$ & $P>0.05$ \\
\hline HLA-DR & $1.26 \pm 1.07$ & $1.91 \pm 1.46$ & $P>0.05$ \\
\hline
\end{tabular}

Data are expressed as mean \pm SD

\section{Mixed lymphocyte culture assays}

Both UCMSCs and AMMSCs at passage 5 were treated with mitomycin $C(50 \mu \mathrm{g} / \mathrm{ml}$ for $60 \mathrm{~min})$ to inhibit the proliferation. $1 \times 10^{4}$ UCMSCs or AMMSCs were co-cultured with $4 \times 10^{4}$ human peripheral blood mononuclear cells (PBMCs) in 96-well culture plate with $1 \mu \mathrm{g} /$ $\mathrm{ml}$ anti-CD3, anti-CD28 and interleukin-2 (Gibco, Grand Island, NY, USA) for 48, 72 and $96 \mathrm{~h}$. Cell Counting Kit-8 assay (Dojindo, Japan) was performed according to the manufacturer's instruction.

\section{Statistical analysis}

Data were expressed as the means \pm SD. Statistical analysis was performed with Student's t-test or One Way ANOVA to compare differences between groups. A $P$ value less than 0.05 was considered statistically significant.

\section{Results \\ Platelet lysis released sufficient amounts of growth factors}

The results showed that PPP contained a certain concentration of PDGF, bFGF, EGF, TGF- $\beta 1$, IGF-1 and VEGF, and freeze-thaw rupture of platelets had greatly elevated levels of growth factors in PL (bFGF and IGF-1, both $P<0.05$; PDGF, EGF, and VEGF, all $P<0.01$; TGF- $\beta 1, P<0.005$; Fig. 1b).

\section{AMMSCs in PL showed similar morphology with UCMSCs}

The UCMSCs and ATMSCs expanded in PL showed fibroblast-like morphologies with parallel or vortex-like patterns, and no morphologic difference was observed between AMMSCs and UCMSCs at passage 5 (Fig. 2a).

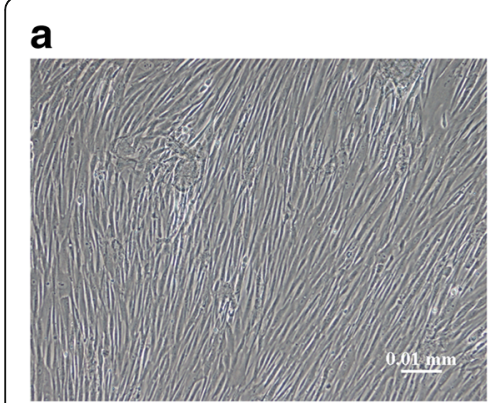

UCMSCs

C

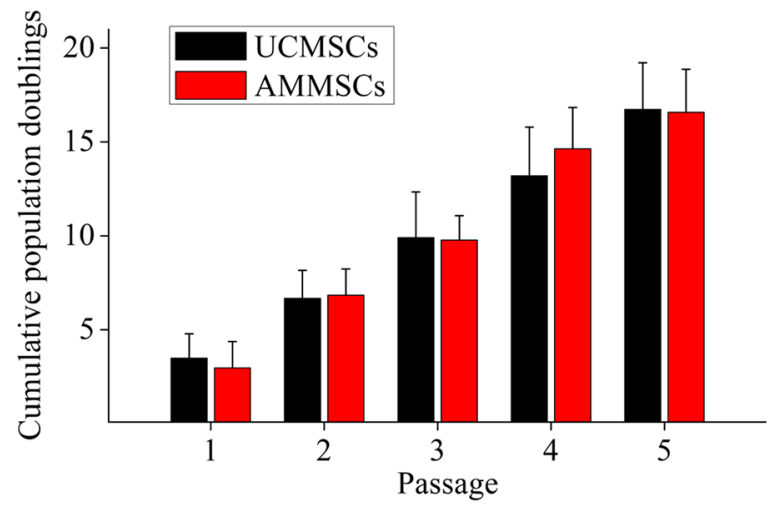

\section{b}

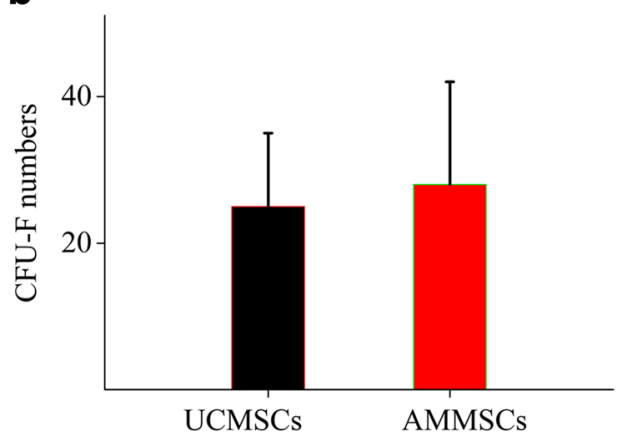

d

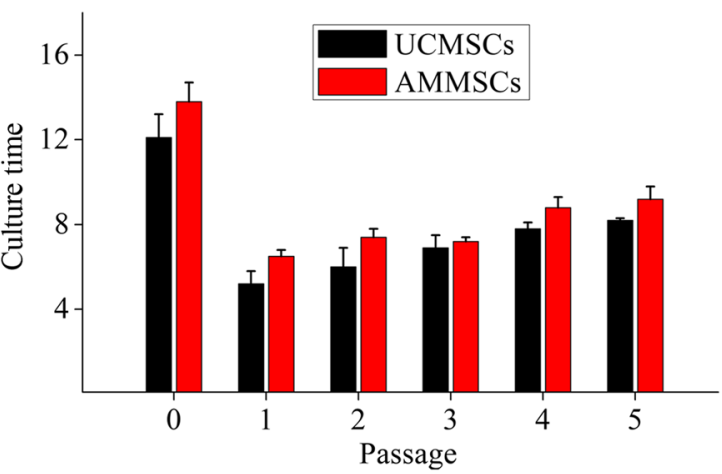

Fig. 2 AMMSCs in PL showed similar morphology and self-renewal capacity with UCMSCs. a Both UCMSCs and ATMSCs expanded in PL showed fibroblast-like morphologies. Scale bar: $10 \mu \mathrm{m}$. b Clone-forming ability of UCMSCs and AMMSCs was assessed by CFU-F counts per $1 \times 10^{5}$ TNCs. The proliferation capacity of UCMSCs and AMMSCs was assessed by CPD $\mathbf{c}$ and culture time $\mathbf{d}$ of each passage. Bars represented means $\pm \mathrm{SD} ; n=5$ 
AMMSCs in PL showed similar self-renewal capacity with UCMSCs

CFU-F analysis showed no significant difference in colony counts between AMMSCs and UCMSCs $(P>0.05$, Fig. 2b). CPD analysis showed that AMMSCs possessed similar CPD numbers (all $P>0.05$, Fig. 2c) and culture times (all $P>0.05$, Fig. $2 \mathrm{~d}$ ) for each passage with UCMSCs, indicating that AMMSCs had similar self-renewal capacity with UCMSCs.

\section{AMMSCs in PL showed similar immunophenotype with UCMSCs}

Flow cytometry analysis showed that both UCMSCs and AMMSCs expanded in PL, expressed high levels of CD73, CD90 and CD105 and lacked expression of CD14, CD19, CD34, CD45 and HLA-DR surface markers. Moreover, there were no significant differences between AMMSCs and UCMSCs (all $P>0.05$, Table 1 ).

\section{AMMSCs in PL showed similar trilineage differentiation potential with UCMSCs}

The osteogenic differentiation of AMMSCs and UCMSCs was confirmed by alizarin red staining of mineralization (Fig. 3a). The osteogenic AMMSCs exhibited a statistically similar mRNA expression of RUNX-2 and alkaline phosphatase with UCMSCs, indicating that AMMSCs in PL had similar osteogenic differentiation potential with UCMSCs (Both $P>0.05$, Fig. 3b).

The chondrogenic differentiation of AMMSCs and UCMSCs was confirmed by alcian blue staining of glycosaminoglycans (Fig. 4a). The chondrogenic AMMSCs exhibited statistically similar mRNA expression of SOX-9 and collagen II with UCMSCs, indicating that AMMSCs in PL had similar chondrogenic differentiation potential with UCMSCs (Both $P>0.05$, Fig. $4 \mathrm{~b}$ ).

The adipogenic differentiation of AMMSCs and UCMSCs was confirmed by oil red $\mathrm{O}$ staining of lipid vacuoles (Fig. 5a). The adipogenic AMMSCs exhibited statistically similar mRNA expression of PPARg and LPL with UCMSCs, indicating that AMMSCs in PL had similar adipogenic differentiation potential with UCMSCs (both $P>0.05$, Fig. 5b).

\section{AMMSCs in PL showed superior immunosuppression effects with UCMSCs}

When no induction, both UCMSCs and AMMSCs exhibited a certain concentration of immunosuppression related mediators, and no significant differences in $\mathrm{PGE}_{2}$

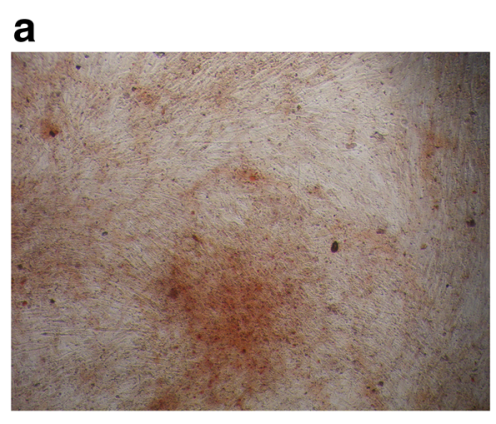

UCMSCs

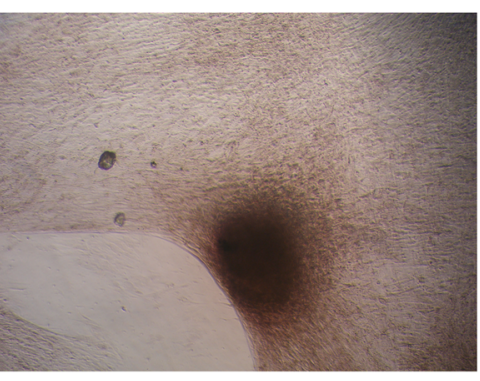

AMMSCs

b

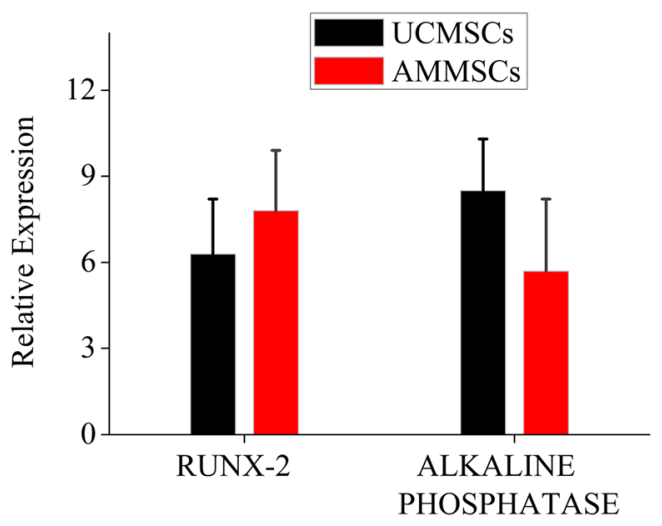

Fig. 3 AMMSCS in PL showed similar osteogenic differentiation potential with UCMSCs. a The osteogenic differentiation of AMMSCS and UCMSCS was confirmed by Alizarin Red staining of mineralization. Magnification $\times 100$. b Comparative investigation of osteogenic differentiation capability of UCMSCs and AMMSCs was assessed by quantitative analysis of RUNX-2 and alkaline phosphatase mRNA expression. Bars represented means $\pm S D ; n=5$ 


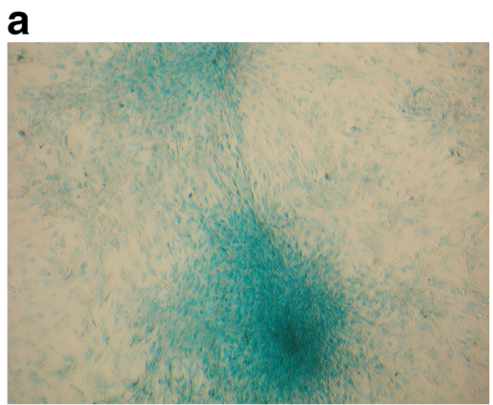

UCMSCs

b

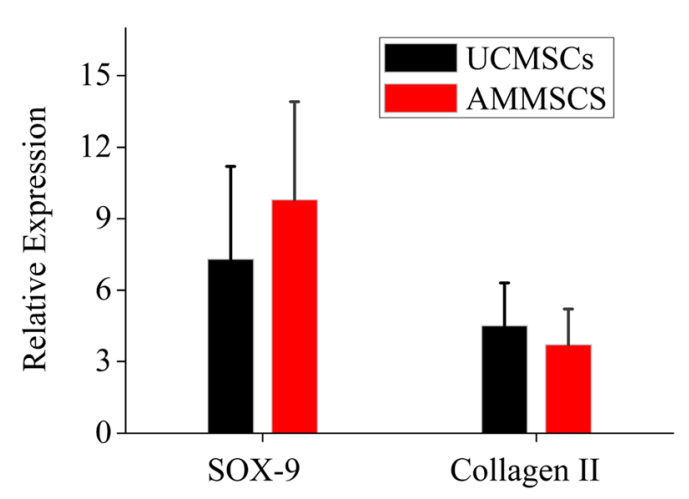

Fig. 4 AMMSCs in PL showed similar chondrogenic differentiation potential with UCMSCs. a The chondrogenic differentiation of AMMSCs and UCMSCs was confirmed by Alcian Blue staining of glycosaminoglycans. Magnification $\times 100$. b Comparative investigation of chondrogenic differentiation capability of UCMSCs and AMMSCs was assessed by quantitative analysis of SOX-9 and collagen II mRNA expression. Bars represented means $\pm \mathrm{SD} ; n=5$

and TGF- $\beta 1$ expression (both $P>0.05$, Fig. 6 a and $b$ ) and IDO activity $(P>0.05$, Fig. $6 \mathrm{c})$ were observed between both types of cells. When primed by IFN- $\gamma$ and (or) TNF- $\alpha$ induction, $\mathrm{PGE}_{2}$ and TGF- $\beta 1$ expression levels and IDO activity were increased in UCMSCs and AMMSCs, and AMMSCs exhibited higher $\mathrm{PGE}_{2}$ expression (all $P<0.05$, Fig. 6a) and IDO activity (all $P<0.05$, Fig. 6c), but similar TGF- $\beta 1$ expression (all $P>0.05$, Fig. 6b) compared with UCMSCs. Moreover, AMMSCs showed a higher inhibitory effect on PBMCs proliferation than UCMSCs at different MSCs/PBMCs ratios (all $P<0.05$, Fig. 6d). These results demonstrated that AMMSCs in PL had superior immunosuppression effects with UCMSCs.

\section{Discussion}

Great success has been reported that PL is an increasingly alternative to FBS as a medium supplement for clinical grade expansion of MSCs for therapeutic applications $[3,20]$. The growth factors including PDGF, TGF- $\beta 1$, EGF and bFGF have been described as mitogens for MSCs [21], and bFGF and PDGF in PL are essential components for the growth-promoting effect of MSCs [22]. Our results show that PPP contains a certain concentration of these growth factors, but could not isolate and expand UCMSCs or AMMSCs in our previous studies (data not shown), demonstrating that low levels of these growth factors are not sufficient for MSCs proliferation. Freeze-thaw rupture of platelets had significantly elevated levels of growth factors in PL, which allowed expansion of UCMSCs and AMMSCs. These results demonstrate that the high concentrations of growth factors in PL are necessary for the proliferation of MSCs. Moreover, these growth factors in synergy with each other promote the proliferation of MSCs. Similar results were already described by others, although the concentrations reported by the various groups differ substantially [23-25]. It is known there is a homogenization of MSCs along time in culture, especially the method without cell sorting. In this study, both UCMSCs and AMMSCs exhibit heterogeneous morphology with various shapes in the early passages, showing low purity. The possibility of some resting mature cells layer in the initial adherent cells and the successive passages leads to a large amount of pure MSCs. However, due to genetic stability in culture, it is recommended by regulatory authorities not to use MSCs from late passages [26]. Moreover, we also found that aging cells appear after passage 5 in a previous study. Therefore, we suggest that MSC at passage 5 is used for evaluation of their characteristics. Similar plot was reported in previous studies [3, 19, 27]. 


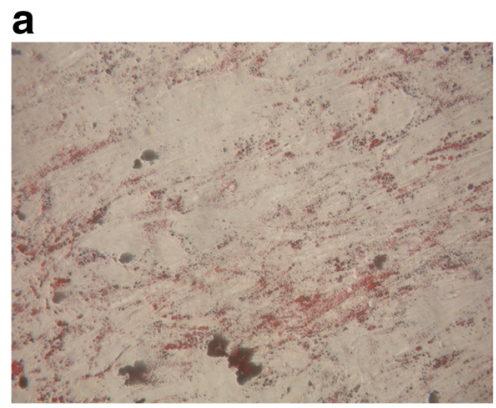

UCMSCs

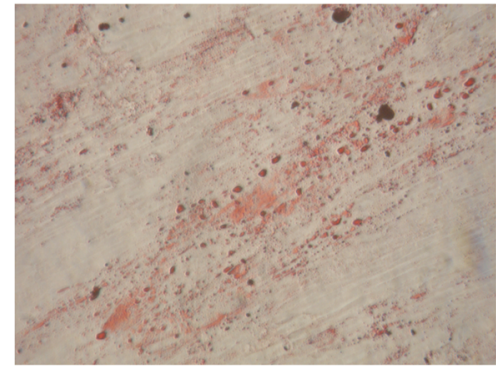

AMMSCs

\section{b}

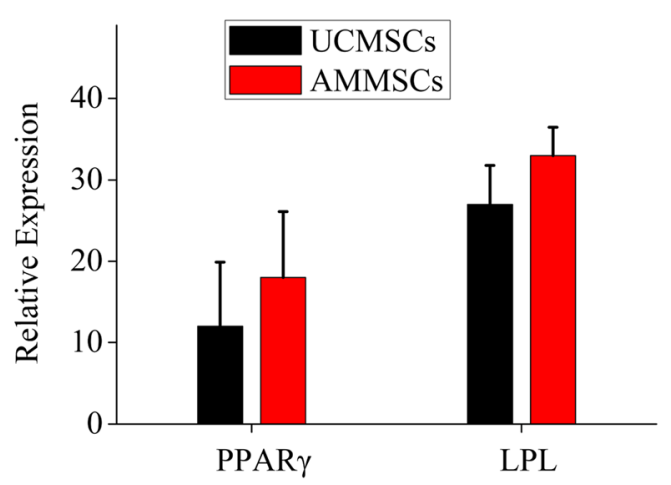

Fig. 5 AMMSCs in PL showed similar adipogenic differentiation potential with UCMSCs. a The adipogenic differentiation of AMMSCS and UCMSCs was confirmed by Oil Red O staining of lipid vacuoles. Magnification $\times 100$. b Comparative investigation of adipogenic differentiation capability of UCMSCs and AMMSCs was assessed by quantitative analysis of PPARg and LPL mRNA expression. Bars represented means $\pm S D ; n=5$

In addition, MSCs-based therapies need viable and ample numbers of MSCs, a patient needs approximately $5 \times 10^{7} \sim 5 \times 10^{8}$ MSCs per transplantation, and usually multiple transplantation. In this study, a sufficient quantity of UCMSCs or AMMSCs can be collected in PL-supplemented cultures in 5 passages at a seeding density of 2000 cells $/ \mathrm{cm}^{2}$. Based on a clinical point of view, passage 5 is more suitable than the earlier passage.

The basic biological characteristics including morphology, phenotype and differentiation capacity have been proposed as minimal criteria for defining MSCs by the International Society for Cellular Therapy (ISCT) [28]. Our results show that both UCMSCs and AMMSCs in PL fulfill all criteria, and no significant differences in these characteristics between both types of cells. It has been described that both $\mathrm{UC}$ and AM are derived from the foetus, this may be the reason why AMMSCs show similar biological characteristics with UCMSCs. Similar performance has been demonstrated between the two types of cells in FBS-supplemented medium $[14,15,29,30]$, but significant differences in morphology, phenotype and trilineage differentiation have been also revealed in FBS in some conflict studies [16, 17]. These apparent differences may be due to different isolation methods of MSCs. It has become widely assumed that besides source and culture condition, isolation methods of is critical in determining the characteristics and therapeutic potential of MSCs [13, 31, 32]. The self-renewal capacity is also one accepted characteristic of adherent MSCs in culture. Our results demonstrate that AMMSCs in PL showed similar self-renewal capacity with UCMSCs, consistent with the FBS-based comparative study [17]. Moreover, the cell population doubling time of the UCMSCs and AMMSCs in cultures supplemented with PL is in the range of $25-45 \mathrm{~h}$ based on our calculation, no significantly differences between two types of cells (data not shown). Compared the results with others available in literature, UCMSCs and AMMSCs in PL showed less cell population doubling time than those in FBS (the range of 45-60 h $[16,17]$ ), showing that higher proliferative capacity in PL, respectively. However, a recent study has reported that AMMSCs in FBS show significantly lower proliferation than UCMSCs $[14,16]$. This different proliferation rates are related to different isolation methods or culture condition. In addition, A possible explanation might be that AM includes two different stem cell populations showing distinct biological characteristics [33].

Increasing evidence in animal models of immune diseases supports the notion that the mechanism of functional benefit of MSCs is predominantly dependent on immunosuppression activity [34]. Thus, the ISCT proposes immune functional assays as potency release criterion for MSCs in 2013 and 2016 [35, 36]. When in a 

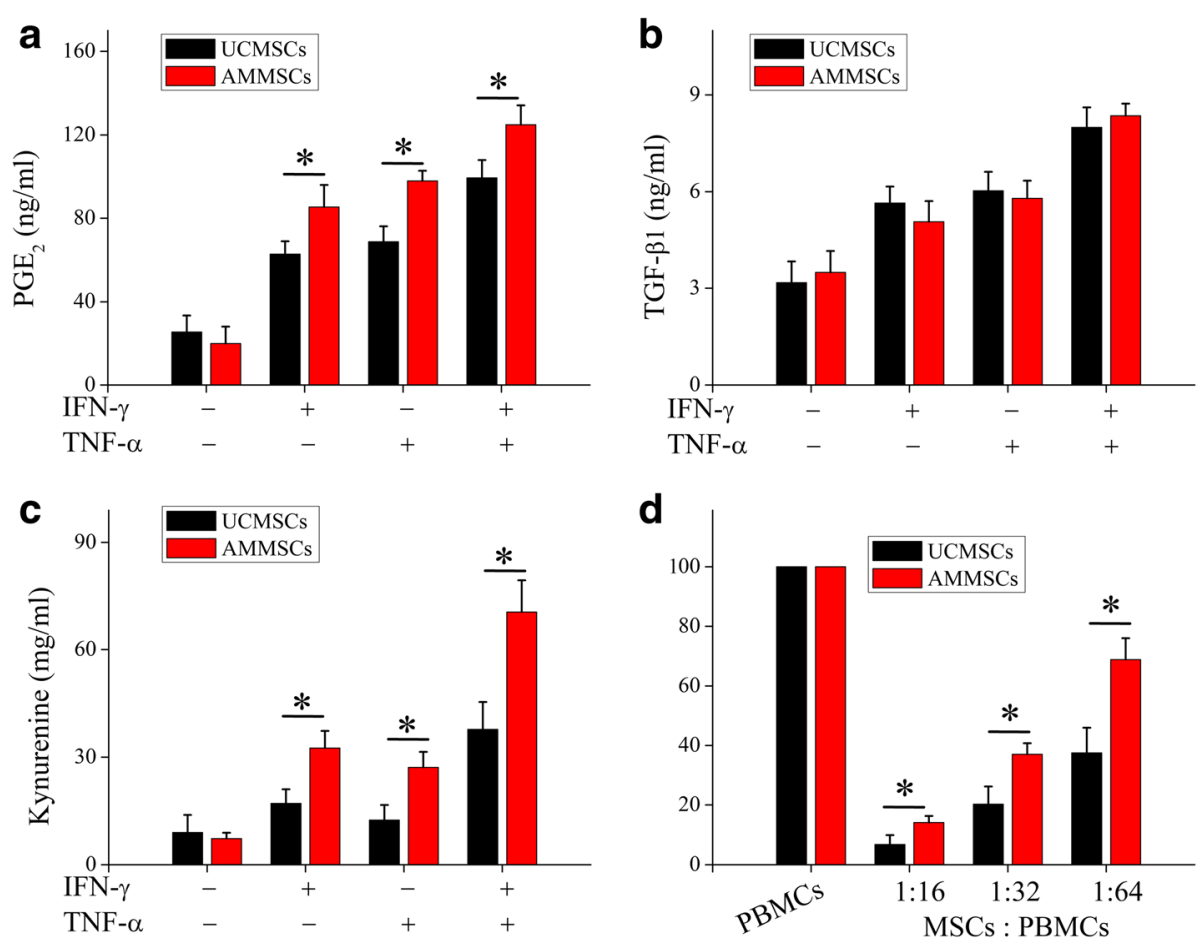

Fig. 6 AMMSCs in PL showed superior immunosuppression effects with UCMSCs. When primed by IFN- $\gamma$ and/or TNF-a induction, PGE 2 (a) and TGF- $\beta 1$ (b) expression were analyzed. IDO activity (c) was evaluated by kynurenine levels. (d) UCMSCs suppressed allogeneic lymphocyte proliferation. Bars represented means $\pm S D, n=5$; ${ }^{*} P<0.05$

pro-inflammatory environment, MSCs could induce anti-inflammatory polarization. Moreover, the activation is necessary for the manifestation of immunosuppressive properties of MSCs. The ISCT suggests that in vitro experimental model with IFN- $\gamma$ and/or TNF- $\alpha$ priming is used as a standard assay for the assessment of immune function of MSCs [35]. In-vitro inflammatory priming of MSCs could, to a certain extent, mimic what happens in vivo when MSCs are transplanted into patients with immune disorders [37]. It has become widely accepted that the addition of IFN- $\gamma$ with or without TNF- $\alpha$ for 12-48 $\mathrm{h}$ is adequate to activate MSCs for the analysis of immunosuppression effect, which is mainly through the secretion of immunosuppression related mediators such as TGF- $\beta 1, \mathrm{PGE}_{2}$ and IDO [34, 37]. Interestingly, our result shows that when no induction, similar levels of $\mathrm{PGE}_{2}$ and TGF- $\beta 1$ expression and IDO activity are observed between UCMSCs and AMMSCs in PL, which is in conflict with the FBS-based comparative analysis described recently $[17,29]$. One study has showed that AMMSCs are significantly higher production of TGF- $\beta$ compared to UCMSCs [25], but another study shows opposite result [23]. These apparent differences may be due to different isolation methods and (or) culture condition of MSCs. In short, both UCMSCs and AMMSCs are to some extent naturally immunosuppressive capabilities without the need for priming, but independent of the culture conditions. Similar capabilities of UCMSCs in FBS-supplemented media have been also demonstrated by assessing anti-inflammatory cytokines in a recent study [38]. We also demonstrate that the priming of UCMSC and AMMSCs leads to an increase in the expression levels of immunosuppression related mediators, showing that the pre-activation could improve the immunosuppression capacity of two types of cells. When primed by IFN- $\gamma$ and (or) TNF- $\alpha$ induction, AMMSCs exhibit higher $\mathrm{PGE}_{2}$ expression and IDO activity than UCMSCs, showing that AMMSCs are more potent immunosuppressors than UCMSCs. This result is in agreement with FBS-based comparison in previous reports $[39,40]$, but in contrast with data from other laboratory [41]. However, similar TGF- $\beta 1$ expression is observed between both types of cells, this may be related to the high level of TGF- $\beta 1$ in PL (Fig. 1b). Importantly, our result further demonstrates that AMMSCs are more potent immunosuppressors by inhibiting PBMCs proliferation effect, which contradicts the FBS-based comparative studies [14]. A possible explanation might be that AMMSCs in FBS are the most heterogeneous population due to low proliferative rate [16]. The high proliferative rate of AMMSCs in PL could lead to a reduction in proportion of some resting epithelial cells within the MSCs layer, and obtain a large amount of pure AMMSCs after the culture of successive passages. 


\section{Conclusions}

In summary, we conduct a direct head-to-head comparison with regard to biological characteristics and immunosuppression effects of AMMSCs and UCMSCs expanded in PL. Our results indicate that AMMSCs in PL have similar morphology, immunophenotype, self-renewal capacity, and trilineage differentiation potential, but superior immunosuppression effects in comparison with UCMSCs. Therefore, we hypothesize that AMMSCs might be more suitable than UCMSCs for treatment of immune diseases. Furthermore, in vivo functional studies are needed to confirm the prediction.

\section{Additional file}

Additional file 1: Primer sequences for Real Time PCR analysis and their respective product sizes. (DOCX $13 \mathrm{~kb}$ )

\begin{abstract}
Abbreviations
AM: Amniotic membrane; AMMSCs: Amniotic membrane derived messechymal stem cells; bFGF: Basic fibroblast growth factor; CFU-F: Colonyforming unit fibroblast; CPD: Cumulative population doublings; EGF: Epithelial growth factor; ELISA: Enzyme linked immunosorbent assay; FBS: Fetal bovine serum; IDO: Indoleamine 2, 3-dioxygenase; IFNY: Interferon-gamma; IGF-1: Insulin-like growth factor-1; IMDM: Iscove's modified dulbecco's medium; ISCT: The International Society for Cellular Therapy; MSCs: Mesenchymal stem cells; PBMCs: Peripheral blood mononuclear cells; PBS: Phosphate buffered saline; PCR: Polymerase chain reaction; PD: Population doubling; PDGF: Platelet-derived growth factor; $\mathrm{PGE}_{2}$ : Prostaglandin $\mathrm{E}_{2} ;$ PL: Platelet lysate; PPP: Platelet-poor plasma; PRP: Platelet-rich plasma; SD: Standard deviation; TGF- $\beta 1$ : Transforming growth factor-beta 1; TNF-a: Tumor necrosis factor-alpha; UC: Umbilical cord; UCMSCs: Umbilical cord derived messechymal stem cells; VEGF: Vascular endothelial growth factor
\end{abstract}

\section{Acknowledgements}

The authors thank the technical support on cell cultures from Department of Technology, Beijing JingMeng Stem Cell Technology. Co. Ltd.

\section{Funding}

This work was supported by National Natural Science Foundation of China (No. 81860157) and Natural Science Foundation of Inner Mongolia (No. 2017MS0314). The funding body had no role in the design of the study and collection, analysis, and interpretation of data or in writing the manuscript.

\section{Availability of data and materials}

The datasets used and analysed during the current study are available from the corresponding author on reasonable request.

\section{Authors' contributions}

All authors participated in the design, interpretation of the results, and review of the manuscript; YXM and XYW were involved in the experimentation, and wrote the manuscript; XYW and ZMH were involved in the analysis of the data. All authors read and approved the final manuscript.

\section{Ethics approval and consent to participate}

All human samples were obtained from healthy donors that provided an informed, written consent for research use, and the study was approved by the Ethics Committee of the First Affiliated Hospital of Baotou Medical College.

\section{Consent for publication}

Not applicable.

\section{Competing interests}

The authors declare that they have no competing interests.

\section{Publisher's Note}

Springer Nature remains neutral with regard to jurisdictional claims in published maps and institutional affiliations.

\section{Author details}

${ }^{1}$ Department of Rheumatology, the First Affiliated Hospital of Xi'an Jiaotong University, Xi'an, Shaanxi Pvovince, China. ${ }^{2}$ Department of Interventional Treatment, the First Affiliated Hospital of Baotou Medical College, Inner Mongolia University of Science and Technology, Baotou, Inner Mongolia, China. ${ }^{3}$ Department of Technology, Stem Cell Medicine Engineering \& Technology Research Center of Inner Mongolia, Huhhot, Inner Mongolia, China. ${ }^{4}$ Department of Research and Development, Beijing Jingmeng Stem Cell Technology. Co. Ltd., Beijing, China.

Received: 1 September 2018 Accepted: 28 November 2018 Published online: 13 December 2018

\section{References}

1. Squillaro T, Peluso G, Galderisi U. Clinical trials with mesenchymal stem cells: an update. Cell Transplant. 2016;25:829-48.

2. Solomon J, Csontos L, Clarke D, Bonyhadi M, Zylberberg C, McNiece I, et al. Current perspectives on the use of ancillary materials for the manufacture of cellular therapies. Cytotherapy. 2016;18:1-12.

3. Capelli C, Domenghini M, Borleri G, Bellavita P, Poma R, Carobbio A, et al. Human platelet lysate allows expansion and clinical grade production of mesenchymal stromal cells from small samples of bone marrow aspirates or marrow filter washouts. Bone Marrow Transplant. 2007;40:785-91.

4. Hemeda $\mathrm{H}$, Giebel B, Wagner W. Evaluation of human platelet lysate versus fetal bovine serum for culture of mesenchymal stromal cells. Cytotherapy. 2014;16:170-80.

5. Sharma RR, Pollock K, Hubel A, McKenna D. Mesenchymal stem or stromal cells: a review of clinical applications and manufacturing practices. Transfusion. 2014:54:1418-37.

6. Samsonraj RM, Raghunath M, Nurcombe V, Hui JH, van Wijnen AJ, Cool SM. Concise review: multifaceted characterization of human mesenchymal stem cells for use in regenerative medicine. Stem Cells Transl Med. 2017;6: 2173-85.

7. Lindenmair A, Hatlapatka T, Kollwig G, Hennerbichler S, Gabriel C, Wolbank $S$, et al. Mesenchymal stem or stromal cells from amnion and umbilical cord tissue and their potential for clinical applications. Cell. 2012;1:1061-88.

8. Hass R, Kasper C, Bohm S, Jacobs R. Different populations and sources of human mesenchymal stem cells (MSC): a comparison of adult and neonatal tissue-derived MSC. Cell Commun Signal. 2011;9:12.

9. Wang S, Mundada L, Colomb E, Ohye RG, Si MS. Mesenchymal stem/stromal cells from discarded neonatal sternal tissue: in vitro characterization and Angiogenic properties. Stem Cells Int. 2016;2016:5098747.

10. Ghasemzadeh M, Hosseini E, Ahmadi M, Kamalizad M, Amirizadeh N. Comparable osteogenic capacity of mesenchymal stem or stromal cells derived from human amnion membrane and bone marrow. Cytotechnology. 2018;70:729-39.

11. El Omar R, Beroud J, Stoltz JF, Menu P, Velot E, Decot V. Umbilical cord mesenchymal stem cells: the new gold standard for mesenchymal stem cell-based therapies? Tissue Eng Part B Rev. 2014;20:523-44.

12. Davies JE, Walker JT, Keating A. Concise review: Wharton's jelly: the rich, but enigmatic, source of mesenchymal stromal cells. Stem Cells Transl Med. 2017:6:1620-30.

13. Bortolotti F, Ukovich L, Razban V, Martinelli V, Ruozi G, Pelos B, et al. In vivo therapeutic potential of mesenchymal stromal cells depends on the source and the isolation procedure. Stem Cell Reports. 2015;4:332-9.

14. Manochantr S, Up Y, Kheolamai P, Rojphisan S, Chayosumrit M, Tantrawatpan C, et al. Immunosuppressive properties of mesenchymal stromal cells derived from amnion, placenta, Wharton's jelly and umbilical cord. Intern Med J. 2013;43:430-9.

15. Veryasov VN, Savilova AM, Buyanovskaya OA, Chulkina MM, Pavlovich SV, Sukhikh GT. Isolation of mesenchymal stromal cells from extraembryonic tissues and their characteristics. Bull Exp Biol Med. 2014;157:119-24.

16. Araujo AB, Salton GD, Furlan JM, Schneider N, Angeli MH, Laureano AM, et al. Comparison of human mesenchymal stromal cells from four neonatal tissues: amniotic membrane, chorionic membrane, placental decidua and umbilical cord. Cytotherapy. 2017;19:577-85. 
17. Wu M, Zhang R, Zou Q, Chen Y, Zhou M, Li X, et al. Comparison of the biological characteristics of mesenchymal stem cells derived from the human placenta and umbilical cord. Sci Rep. 2018:8:5014.

18. Murphy MB, Blashki D, Buchanan RM, Yazdi IK, Ferrari M, Simmons PJ, et al. Adult and umbilical cord blood-derived platelet-rich plasma for mesenchymal stem cell proliferation, chemotaxis, and cryo-preservation. Biomaterials. 2012;33:5308-16.

19. Li CY, Wu XY, Tong JB, Yang XX, Zhao JL, Zheng QF, et al. Comparative analysis of human mesenchymal stem cells from bone marrow and adipose tissue under xeno-free conditions for cell therapy. Stem Cell Res Ther. 2015;6:55.

20. Tarte K, Gaillard J, Lataillade JJ, Fouillard L, Becker M, Mossafa H, et al. Clinical-grade production of human mesenchymal stromal cells: occurrence of aneuploidy without transformation. Blood. 2010;115:1549-53.

21. Ng F, Boucher S, Koh S, Sastry KS, Chase L, Lakshmipathy U, et al. PDGF, TGF-beta, and FGF signaling is important for differentiation and growth of mesenchymal stem cells (MSCs): transcriptional profiling can identify markers and signaling pathways important in differentiation of MSCs into adipogenic, chondrogenic, and osteogenic lineages. Blood. 2008;112:295-307.

22. Fekete N, Gadelorge M, Furst D, Maurer C, Dausend J, Fleury-Cappellesso S, et al. Platelet lysate from whole blood-derived pooled platelet concentrates and apheresis-derived platelet concentrates for the isolation and expansion of human bone marrow mesenchymal stromal cells: production process, content and identification of active components. Cytotherapy. 2012:14:540-54

23. Doucet C, Ernou I, Zhang Y, Llense JR, Begot L, Holy X, et al. Platelet lysates promote mesenchymal stem cell expansion: a safety substitute for animal serum in cell-based therapy applications. J Cell Physiol. 2005;205:228-36.

24. Weibrich G, Kleis WK, Hafner G, Hitzler WE. Growth factor levels in plateletrich plasma and correlations with donor age, sex, and platelet count. J Craniomaxillofac Surg. 2002;30:97-102.

25. Eppley BL, Woodell JE, Higgins J. Platelet quantification and growth factor analysis from platelet-rich plasma: implications for wound healing. Plast Reconstr Surg. 2004;114:1502-8.

26. Barkholt L, Flory E, Jekerle V, Lucas-Samuel S, Ahnert P, Bisset $L$, et al. Risk of tumorigenicity in mesenchymal stromal cell-based therapies--bridging scientific observations and regulatory viewpoints. Cytotherapy. 2013;15:753-9.

27. Jin HJ, Bae YK, Kim M, Kwon SJ, Jeon HB, Choi SJ, et al. Comparative analysis of human mesenchymal stem cells from bone marrow, adipose tissue, and umbilical cord blood as sources of cell therapy. Int J Mol Sci. 2013;14: 17986-8001.

28. Dominici M, Le Blanc K, Mueller I, Slaper-Cortenbach I, Marini F, Krause D, et al. Minimal criteria for defining multipotent mesenchymal stromal cells. The International Society for Cellular Therapy position statement. Cytotherapy. 2006:8:315-7.

29. Dabrowski FA, Burdzinska A, Kulesza A, Sladowska A, Zolocinska A, Gala K, et al. Comparison of the paracrine activity of mesenchymal stem cells derived from human umbilical cord, amniotic membrane and adipose tissue. J Obstet Gynaecol Res. 2017:43:1758-68.

30. Ertl J, Pichlsberger M, Tuca AC, Wurzer P, Fuchs J, Geyer SH, et al. Comparative study of regenerative effects of mesenchymal stem cells derived from placental amnion, chorion and umbilical cord on dermal wounds. Placenta. 2018;65:37-46.

31. Corotchi MC, Popa MA, Remes A, Sima LE, Gussi I, Lupu Plesu M. Isolation method and xeno-free culture conditions influence multipotent differentiation capacity of human Wharton's jelly-derived mesenchymal stem cells. Stem Cell Res Ther. 2013;4:81.

32. Hua J, Gong J, Meng H, Xu B, Yao L, Qian M et al. Comparison of different methods for the isolation of mesenchymal stem cells from umbilical cord matrix: proliferation and multilineage differentiation as compared to mesenchymal stem cells from umbilical cord blood and bone marrow. Cell Biol Int. 2014:38:198-210.

33. Ding $\mathrm{C}$, Li H, Wang Y, Wang F, Wu H, Chen R, et al. Different therapeutic effects of cells derived from human amniotic membrane on premature ovarian aging depend on distinct cellular biological characteristics. Stem Cell Res Ther. 2017;8:173.

34. Lee HK, Lim SH, Chung IS, Park Y, Park MJ, Kim JY, et al. Preclinical efficacy and mechanisms of mesenchymal stem cells in animal models of autoimmune diseases. Immune Netw. 2014;14:81-8.

35. Krampera M, Galipeau J, Shi Y, Tarte K, Sensebe L, MSCCotISfC T. Immunological characterization of multipotent mesenchymal stromal cells--the International Society for Cellular Therapy (ISCT) working proposal. Cytotherapy. 2013;15:1054-61.
36. Galipeau J, Krampera M, Barrett J, Dazzi F, Deans RJ, DeBruijn J, et al. International Society for Cellular Therapy perspective on immune functional assays for mesenchymal stromal cells as potency release criterion for advanced phase clinical trials. Cytotherapy. 2016;18:151-9.

37. de Wolf C, van de Bovenkamp M, Hoefnagel M. Regulatory perspective on in vitro potency assays for human mesenchymal stromal cells used in immunotherapy. Cytotherapy. 2017;19:784-97.

38. Barcia RN, Santos JM, Filipe M, Teixeira M, Martins JP, Almeida J, et al. What makes umbilical cord tissue-derived mesenchymal stromal cells superior Immunomodulators when compared to bone marrow derived mesenchymal stromal cells? Stem Cells Int. 2015;2015:583984.

39. Talwadekar MD, Kale VP, Limaye LS. Placenta-derived mesenchymal stem cells possess better immunoregulatory properties compared to their cordderived counterparts-a paired sample study. Sci Rep. 2015;5:15784.

40. Yamahara K, Harada K, Ohshima M, Ishikane S, Ohnishi S, Tsuda H, et al. Comparison of angiogenic, cytoprotective, and immunosuppressive properties of human amnion- and chorion-derived mesenchymal stem cells. PLoS One. 2014;9:e88319.

41. Chen G, Yue A, Ruan Z, Yin Y, Wang R, Ren Y, et al. Comparison of biological characteristics of mesenchymal stem cells derived from maternal-origin placenta and Wharton's jelly. Stem Cell Res Ther. 2015;6:228.

\section{Ready to submit your research? Choose BMC and benefit from:}

- fast, convenient online submission

- thorough peer review by experienced researchers in your field

- rapid publication on acceptance

- support for research data, including large and complex data types

- gold Open Access which fosters wider collaboration and increased citations

- maximum visibility for your research: over $100 \mathrm{M}$ website views per year

At $\mathrm{BMC}$, research is always in progress.

Learn more biomedcentral.com/submissions 